\title{
Antinuclear antibodies in healthy people: the tip of autoimmunity's iceberg?
}

\author{
David S Pisetsky ${ }^{1,2}$ \\ See related research by Li et al., http://arthritis-research.com/content/13/2/R38
}

\begin{abstract}
Antinuclear antibodies (ANAs) are venerable

biomarkers for assessing the diagnosis and prognosis

of patients with autoimmunity. While closely associated

with diseases such as systemic lupus erythematosus,

ANA expression occurs commonly in healthy people.

The basis for this expression is unknown, although

it may reflect features of the assays for antibody

detection or intrinsic immunological disturbances in

otherwise normal individuals. Like autoimmunity itself,

ANA expression is more common among women than

men, pointing to an important determinant of these

responses. Future research will clarify the mechanisms

of ANA expression and the utility of current assays as

antecedent and screening biomarkers.
\end{abstract}

Throughout medicine, investigators are in hot pursuit of biomarkers. These biomarkers, many of which involve multiplex assays or 'omic' technologies, come in a variety of flavors: antecedent (disease risk); screening (subclinical disease); diagnostic; staging; and prognostic. Among their uses, biomarkers can help elucidate genetic predisposition to disease and identify triggering events; practically, such markers can allow early diagnosis and treatment and the development of strategies for risk reduction. Although biomarker technology can be unbelievably complex, the principles are straightforward and provide hope for improved patient outcomes.

As the study by Li and colleagues in Arthritis Research and Therapy indicates [1], the use of antinuclear antibodies (ANAs), one of the most venerable tests in immunology, as antecedent or screening biomarkers, while potentially very informative, faces major challenges.

*Correspondence: piset001@mc.duke.edu

'Medical Research Service, Durham Veterans Administration Medical Center,

508 Fulton St, Durham, NC 27705, USA

Full list of author information is available at the end of the article
Amongst these, the frequency of serological positivity in the general population is probably the greatest. While the actual frequency of positive assays varies with methodology, nevertheless, up to $20 \%$ or more of otherwise healthy people can express an ANA [2]. The expression of these antibodies does not appear related to age despite ideas that immunosenescence may promote autoreactivty [1].

The basis of this seropositivity is puzzling. One possibility is that ANA reactivity represents vagaries of the assays, allowing detection of antibodies of either low titer or low avidity. Many nuclear antigens are highly charged molecules, with DNA and histones the prime examples. As such, ANA binding may occur by chargecharge interactions or cross-reactivity with other antigens (also charged). In this regard, solid phase or multiplex assays may reveal a different perspective on serology than the classic (and now antiquated) methods. These older assays required large amounts of antibody for detection, such as the formation of precipitating complexes in immunodiffusion assays [3]. As a result, seropositivity indicated a robust response. While the solid phase and multiplex assays are sensitive and allow high throughput, their interpretation requires caution, especially in the setting of preclinical or subclinical disease, where the measured responses may be low $[4,5]$.

Another explanation for the frequency of ANA expression in the general population relates to intrinsic immunological disturbances among humans. Perhaps as a species, humans are predisposed to autoimmunity, with ANA expression the tip of the iceberg of autoimmunity. In animal models, ANA production can occur in the absence of other manifestations of systemic lupus erythematosus, reflecting the actions of specific genes that promote immune cell activity. While studies in mice involve intentional efforts to isolate genes for autoimmunity, the human genome may nevertheless contain numerous polymorphisms to increase species fitness to fight off infection or heal wounds [6]. Indeed, the selective pressure created by infection can be profound, with the evolution of genes for nitric oxide production, for example, implicated in a predisposition to diseases 
such as lupus and rheumatoid arthritis as well as defense against malaria [7]. Certainly, more extensive analysis of the serology of various racial and ethnic groups would be informative, as would the study of populations in other locales [8].

As shown in this and other studies, ANA reactivity is greater in women than men, although these gender differences did not occur with antibodies to citrullinated proteins. In an era of genetics and personalized medicine, the biological differences between women and men sometimes do not get the attention they deserve. While the role of hormones compared to the genetic endowment of two Xs versus an XY tandem can be debated, nevertheless, women appear predisposed to lupus as well as baseline ANA reactivity. In the future, consideration of the role of pregnancy in ANA reactivity seems worthwhile since, during normal pregnancy, there can be extensive exposure to nuclear antigens. Furthermore, although often considered a time of immunosuppression, pregnancy actually shows a surge of inflammatory activity that could impact on immune responsiveness $[9,10]$.

As almost every study has demonstrated, lupus is an enormously complex condition, with each patient displaying a seemingly unique set of immunological disturbances and clinical and serological manifestations. In this circumstance, the chances of finding antecedent markers, including gene signatures, may be limited. Indeed, in the current study, the array studies produced surprising results since some healthy subjects without autoantibodies had greater gene up-regulation than those with autoantibodies [1]. More work will be needed to understand the interplay between gene expression and serology as well as the determinants of the interferon signature, which has been linked to immune complexes composed of ANAs.

In the real world of patient care, when confronting a positive ANA in a patient without clinical disease but consistent symptoms, the physician and patient want to know who will develop lupus and who will be spared, whether by luck, happenstance or even preventative measures such as very early therapy. The road to that point will be long but the study by $\mathrm{Li}$ and colleagues is a very promising start of the journey.

\section{Abbreviations}

ANA, antinuclear antibody.

\section{Competing interests}

The author declares that he has no competing interests.

\section{Acknowledgements}

These studies were supported by a VA Merit Review grant and NIH Grant Al082402.

\section{Author details}

'Medical Research Service, Durham Veterans Administration Medical Center, 508 Fulton St, Durham, NC 27705, USA. ²Division of Rheumatology and Immunology, Duke University Medical Center, Durham, NC 27705, USA.

Published: 21 April 2011

\section{References}

1. Li Q-Z, Karp DR, Quan, J, Branch VK, Zhou J, Lian Y, Chong BF, Wakeland EK, Olsen NJ: Risk factors for ANA positivity in healthy persons. Arthritis Res Ther 2011, 13:R38.

2. Wandstrat AE, Carr-Johnson F, Branch V, Gray H, Fairhurst A-M, Reimold A, Karp D, Wakeland EK, Olsen NJ: Autoantibody profiling to identify individuals at risk for systemic lupus erythematosus. J Autoimmunity 2006, 27:153-160.

3. Tan EM: Antinuclear antibodies: diagnostic markers for autoimmune diseases and probes for cell biology. Adv Immunol 1989, 44:93-151.

4. Copple SS, Martins TB, Masterson C, Joly E, Hill HR: Comparison of three multiplex immunoassays for detection of antibodies to extractable nuclear antibodies using clinically defined sera. Ann NY Acad Sci 2007, 1109:464-472.

5. Meroni PL, Schur PH: ANA screening: an old test with new recommendations. Ann Rheum Dis 2010, 69:1420-1422.

6. Rai E, Wakeland EK: Genetic predisposition to autoimmunity - what have we learned? Semin Immunol 2011 [Epub ahead of print]

7. Oates JC, Levesque MC, Hobbs MR, Smith EG, Molano ID, Page GP, Hill BS, Weinberg JB, Cooper GS, Gilkeson GS: Nitric oxide synthase 2 promoter polymorphisms and systemic lupus erythematosus in African-americans. J Rheumatol 2003, 30:60-67.

8. Borchers AT, Naguwa SM, Shoenfeld Y, Gershwin ME: The geoepidemiology of systemic lupus erythematosus. Autoimmunity Rev 2010, 9:A277-A287.

9. Sacks GP, Studena K, Sargent IL, Redman CWG: Normal pregnancy and preeclampsia both produce inflammatory changes in peripheral blood leukocytes akin to those of sepsis. Am J Obstet Gynecol 1998, 179:80-86.

10. Bianchi DW, Wataganara T, Lapaire O, Tjoa ML, Maron JL, Larrabee PB, Johnson KL: Fetal nucleic acids in maternal body fluids. Ann NY Acad Sci 2006, 1075:63-73.

doi:10.1186/ar3282

Cite this article as: Pisetsky DS: Antinuclear antibodies in healthy people: the tip of autoimmunity's iceberg? Arthritis Research \& Therapy 2011, 13:109. 